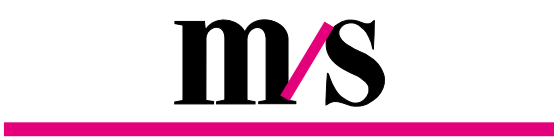

médecinel scien ces $2001 ; 151-4$

\title{
L'ATHÉROSCLÉROSE : UN DÉFI COMMUN DU BIOLOGISTE ET DU CLINICIEN
}

\section{Jacques Bonnet}

\section{ADRESSES}

J. Bonnet : Inserm U. 441, Centre de recherche Inserm, avenue du $\mathrm{H}$ aut-Lévêque, 33600 Pessac Cedex, France. 'athérosclérose est responsable de la plus grande partie des décès dans les pays occidentalisés et apparaît en nette progression dans les pays en voie de développement, comme si cette pathologie était le reflet inéluctable du progrès social. Retrouvée pourtant dès l'Égypte ancienne, elle apparaît comme une maladie dégénérative de l'homme, vraisemblablement responsable depuis toujours, avec les pathologies valvulaires, de la majorité des pathologies cardio-vasculaires. Si les pathologies valvulaires rhumatismales ont aujourd'hui presque disparu des pays développés, l'athérosclérose reste quant à elle toujours présente et toujours aussi meurtrière. Pourtant, au cours de ces dernières années, la recherche fondamentale sur la biologie vasculaire et les métabolismes lipidique et glucidique, la recherche clinique sur les pathologies coronariennes, carotidiennes et artérielles périphériques, les recherches épidémiologiques, les recherches pharmacologiques se sont rassemblées autour de cette pathologie avec un objectif qui est la diminution de l'incidence des pathologies cardio-vasculaires. Les progrès dans la compréhension des étapes majeures de la pathologie ont été considérables et la confrontation de ces disciplines apparaît plus que jamais nécessaire à la réalisation de l'objectif commun.

\section{Le dysfonctionnement endothélial : étape initiale de l'athérosclérose}

La première étape clé de l'athérosclérose est le dysfonctionnement endothélial. Au contact du sang circulant, I'endothélium, soumis à la double contrainte des forces de cisaillement et de la tension pariétale, contrôle la vasomotricité et le profil non-thrombotique du vaisseau. Les grandes fonctions endothéliales et particulièrement la capacité de contrôler la vasodilatation artérielle vont être touchées très précocement, avant même l'apparition des premières lésions artérielles cliniquement détectables. Les causes de ce dysfonctionnement endothélial sont multiples et souvent imparfaitement comprises sur le plan moléculaire: perte du flux laminaire responsable d'une diminution locale des forces de cisaillement et d'une stimulation de la prolifération des cellules endothéliales entraînant l'augmentation de leur perméabilité [1]; présence de dérivés potentiellement toxiques dans l'environnement immédiat de la cellule; excès plasmatique de LDL associé précocement à l'accumulation pariétale de cholestérol; accumulation sous-endothéliale puis intra-cellulaire de particules de LDL natives, légèrement modifiées ou déjà oxydées [2]. La formation de produits d'oxydation joue en effet un 
rôle déterminant dans l'induction du dysfonctionnement endothélial [3,4]. Enfin, il ne faut pas négliger le rôle potentiel des agents infectieux dans la genèse du climat de stress oxydatif de l'endothélium [5].

Les conséquences de ce dysfonctionnement endothélial sont multiples. La première fonction touchée est la capacité de l'endothélium à induire une vasodilatation, essentiellement liée à une diminution du NO endothélial. Les autres fonctions endothéliales de perméabilité et d'adhésivité vont également être touchées. L'augmentation de la perméabilité va favoriser la pénétration des $L D L$, et l'apparition des protéines d'adhérence endothéliale comme VCAM-1 (vascular cell adhesion molecule1), associée à l'expression de protéines chimioattractantes comme le MCP-1 (monocyte chemotactic protein), va permettre le début de l'infiltration pariétale par les cellules inflammatoires.

Les implications cliniques de ce dysfonctionnement endothélial sont importantes et constituent un des axes majeurs de la prévention de l'athérosclérose. Le dysfonctionnement endothélial étant la première étape de l'athérosclérose, le clinicien aborde ce problème d'une part en définissant des méthodes d'analyse de ce dysfonctionnement et en essayant de développer une pharmacologie dont l'objectif thérapeutique est la protection endothéliale. La détection précoce du dysfonctionnement endothélial est difficile en clinique. II peut être identifié par l'étude de la variation d'expression des marqueurs endothéliaux comme les taux plasmatiques de cytokines ou de protéines d'adhérence endothéliales, s( soluble) VCAM-1 ou sICAM-1s (Intercellular cell adhesion molecule), sEsélectine [6]. II est cependant de plus en plus évident qu'une telle approche n'a pas de valeur à l'échelle individuelle. La seule voie d'exploration actuelle reste la mesure directe de la vasomotricité dépendant de l'endothélium sur l'artère brachiale en hyperhémie (méthode assez lourde, difficilement applicable en pratique courante [7]), qui permet d'analyser précocement l'atteinte de cette fonction chez le malade et sa rentes thérapeutiques. II faut enfin noter que la susceptibilité de l'endothélium aux différents facteurs de risque est sûrement la traduction d'un terrain génétique dont l'existence est de plus en plus abordée grâce à l'analyse des polymorphismes génétiques [8]. Sur le plan thérapeutique, plusieurs approches sont actuellement développées avec pour cible la protection endothéliale et particulièrement la récupération de la fonction de vasodilatation endothélium-dépendante. Ainsi, les inhibiteurs de l'enzyme de conversion ou du récepteur AT1, les inhibiteurs de la réductase d'HMG CoA [9], les antioxydants ou autres approches nutritionnelles sont capables de restaurer certaines fonctions endothéliales et donc a priori de retarder ou de ralentir l'évolution de la phase initiale de l'athérosclérose.

\section{Le processus inflammatoire et la progression des lésions athéroscléreuses}

La progression de la lésion est liée à plusieurs phénomènes, l'infiltration massive de cellules inflammatoires, monocytes/ macrophages et lymphocytes, associée à la poursuite de l'infiltration lipidique et à la formation de cellules spumeuses, et enfin l'arrivée des cellules musculaires lisses qui vont synthétiser une matrice extra-cellulaire plus ou moins abondante et former la chape fibreuse.

La réaction inflammatoire dépend de l'infiltration pariétale des cellules mononucléées d'origine circulante, liée au double processus cellulaire d'adhérence et de migration transendothéliale de ces cellules $[9,10]$. Au cours de ces dernières années, les mécanismes moléculaires contrôlant ces différentes étapes ont été partiellement définis. La conséquence la plus grave de cette inflammation est qu'elle facilite la rupture de plaque, en favorisant la dégradation de la matrice extra-cellulaire par les enzymes protéolytiques, l'induction de l'apoptose des cellules musculaires lisses et le début d'un processus thrombotique par production de facteur tissulaire. Nous reviendrons sur cette complication. L'oxydation minimale ou majeure des LDL piégées dans le sous-endothélium est un des facteurs importants de cette accumulation initiale de cellules mononucléées. L'amplification de la réaction inflammatoire implique ensuite I'interaction entre monocytes/ macrophages et lymphocytes, assurée en partie par les intégrines leucocytaires mais aussi par des systèmes plus directement impliqués dans les pathologies inflammatoires comme les interactions CD 40-CD 40 ligand ou l'équilibre entre cytokines pro- et anti-inflammatoires au sein de la plaque, reflet de l'équilibre entre les lymphocytes Th1 et Th2 [11,13].

L'organisation de la chape fibreuse est le deuxième facteur de développement des plaques athéroscléreuses. Les cellules musculaires lisses vont s'accumuler de façon progressive dans l'intima et encapsuler progressivement le centre nécrotique des cellules spumeuses. L'origine de ces cellules musculaires lisses n'est pas tout à fait claire [14]. L'hypothèse la plus vraisemblable est cependant leur origine médiale. Si les phénomènes de dédifférenciation, de migration et de prolifération des cellules musculaires lisses sont importants dans la constitution de la chape fibreuse, et potentiellement dans la progression de certaines plaques dans lesquelles la composante fibreuse prédomine, l'arrêt de cette progression et la stabilisation des plaques sont liés en partie à l'arrêt de ces processus cellulaires pathologiques [15].

L'implication clinique de la progression des plaques athéroscléreuses est majeure mais le phénomène est difficile à appréhender pour le clinicien. La progression des plaques aboutit à une réduction progressive du calibre artériel dans un territoire donné, avec pour conséquences la réduction des potentialités d'adaptation du débit artériel en condition extrême (effort) dans le territoire considéré et l'induction d'une ischémie. A ce stade tardif, la détection des lésions artérielles est généralement facile par la réalisation de tests fonctionnels. Cependant, un des objectifs du médecin est de détecter la présence et I'évolution de l'athérosclérose avant la survenue de ce stade tardif et 
des complications liées à la rupture de plaque, afin d'assurer une prévention plus efficace qui, à ce stade de la maladie, est toujours une prévention primaire. Dans ce cadre, différents marqueurs utiles en clinique ont été développés. Certains sont le reflet d'une des étapes physiopathologiques du développement de la plaque comme les marqueurs du dysfonctionnement ou de l'activation endothéliale $[16,17]$, de l'oxydation des lipoprotéines [18], ou de I'inflammation telle la $\mathrm{C}$-reactive protein (CRP) ou le dosage des cytokines circulantes, pro-inflammatoires, TNF $\alpha$, IL-1 et IL 6, ou anti-inflammatoires, IL-10 et TGF $\beta$ [19]. Quelle que soit la validité prouvée de ces tests dans les études épidémiologiques, leur intérêt en pratique clinique à l'échelon individuel reste faible. Parallèlement à ces marqueurs biologiques s'est développée une autre approche, centrée sur I'analyse de marqueurs plus intégratifs du risque et de l'atteinte artérielle. La détection précoce de calcifications coronariennes a pu être utilisée, mais le marqueur le plus intéressant semble être I'IMT (intima media thickness), qui permet de détecter les patients à risque vasculaire élevé. L'indice d'IMT [20,21] correspond à la mesure réelle et sommée de l'épaisseur de la média et de l'intima, analysée par échographie au niveau de la carotide commune, artère développant initialement un épaississement dégénératif puis secondairement des plaques fibreuses diffuses. L'autre préoccupation majeure du clinicien est la prise en charge du traitement du patient porteur d'une atteinte athéroscléreuse débutante. L'objectif de cette approche thérapeutique est de contrôler l'évolution des plaques, afin d'éviter leur rupture mais aussi de limiter leur progression et leur retentissement fonctionnel. Au-delà du contrôle de l'évolution de I'hyperplasie intimale et du remodelage adaptatif du vaisseau, objectif thérapeutique essentiel, il existe également deux autres objectifs qui sont les inhibitions des resténoses post-angioplastie et intrastent. Actuellement, aucun traitement n'a pu contrôler directement la réponse pariétale. Cependant, beau- coup de nouvelles stratégies, centrées sur l'utilisation d'anticorps spécifiques, d'inactivation génique spécifique, ou de pharmacologie plus classique, se développent en prenant pour cible soit la réaction inflammatoire pariétale (anti-VCAM-1, antiCD40/ CD40L, anti-cytokines...), soit la réponse musculaire lisse. Ces nouvelles stratégies n'ont pas, pour le moment, dépassé le stade expérimental. Les seules thérapeutiques applicables chez I'homme sont celles centrées sur le contrôle des troubles lipidiques. L'action des inhibiteurs d'HMG CoA reductase et des fibrates apparaît beaucoup plus complexe que leur simple action sur les paramètres lipidiques plasmatiques, et semble également agir directement sur les différentes phases de la réaction inflammatoire.

\section{La rupture de plaque et ses conséquences: le syndrome coronarien aigu}

L'athérosclérose devient vraiment une maladie au stade des lésions compliquées. A un stade plus ou moins tardif de son évolution la plaque va se rompre, conséquence de trois mécanismes: l'évolution en taille et en volume du centre nécrotique, zone avasculaire riche en lipides et sans support collagène [22], I'inflammation et la dégradation matricielle de la chape fibreuse et le défaut de réparation de la chape par les cellules musculaires lisses.

Au niveau coronarien, ceci se traduit par la survenue brutale d'une occlusion totale ou sub-totale de la lumière artérielle entraînant l'apparition d'un syndrome coronarien aigu, c'est à dire un angor instable, un infarctus du myocarde sans onde Q ou un infarctus transmural. Deux tiers des plaques qui rompent ont un degré de sténose inférieur à $50 \%$, et $97 \%$ des patients ont une sténose avant rupture inférieure à $70 \%$ de la lumière artérielle.

La rupture de plaque va être à l'origine du deuxième mécanisme responsable des syndromes coronariens aigus, la thrombose artérielle. La rupture de plaque va être responsable initialement de l'adhérence et de l'activation plaquettaire associées au relargage du contenu des granules plaquettaires, rapidement suivi de l'agrégation plaquettaire. Le thrombus plaquettaire fragile et instable ne peut s'organiser que par l'activation simultanée de la coagulation et la formation de fibrine. Le contact entre le sang circulant et le centre nécrotique, où se sont accumulés les corps apoptotiques et le facteur tissulaire synthétisé et sécrété par les macrophages et les cellules musculaires lisses apparaît comme une étape clé. L'irruption du facteur tissulaire au contact du sang circulant va permettre, par son association avec le facteur VIIa, l'activation de la cascade enzymatique qui conduit à la formation de thrombine et au dépôt de fibrine [23]. Cette activation massive de la coagulation est théoriquement contrôlée par le système fibrinolytique pariétal malheureusement souvent insuffisant pour limiter l'ampleur du phénomène. Cependant, l'évolution du thrombus est dynamique et le contrôle de l'extension du processus thrombotique par la thrombolyse locale physiologique va parfois permettre la stabilisation du syndrome coronarien et stopper l'évolution vers l'infarctus du myocarde.

L'apparition du syndrome coronarien aigu apparaît ainsi comme une étape déterminante dans l'évolution de l'athérosclérose. C'est une crainte permanente du clinicien. Le diagnostic de rupture de plaque au stade du syndrome coronarien aigu est évidemment trop tardif, et un des objectifs majeurs de la recherche est de trouver le moyen de détecter les plaques potentiellement instables. Les marqueurs biologiques, notamment de l'inflammation, restent imparfaits et non spécifiques. En revanche, il est vraisemblable que les nouvelles techniques d'imagerie non invasives, telles que I'IRM et les techniques utilisant les ultrasons, pourront définir la vulnérabilité des plaques par l'analyse qualitative de leurs caractéristiques structurales. Sur le plan thérapeutique, l'approche du syndrome coronarien aigu a été totalement révolutionné au cours des dernières années par le contrôle du processus thrombotique. La mise à la disposition des cardiologues des thé- 
rapeutiques anti-plaquettaires, aspirine, ticlopidine, clopidogrel, antagonistes des récepteurs anti-intégrines plaquettaires GPIIblIIa, associées aux thérapeutiques neutralisant la thrombine (héparine non fractionnée, héparine de bas poids moléculaire, anti-thrombine direct), et aux thérapeutiques fibrinolytiques, a permis de contrôler la formation du thrombus coronarien. Associés aux techniques d'angioplastie, ces traitements permettent de faire de la revascularisation coronarienne précoce l'objectif thérapeutique absolu des syndromes coronariens aigus. La prochaine étape du progrès sera le contrôle des mécanismes de la rupture de plaque. II est vraisemblable que l'efficacité des inhibiteurs de I'HMG CoA réductase dans la prévention primaire et secondaire est partiellement liée au contrôle de certaines étapes cellulaires de la rupture de plaques. Néanmoins, il est évident que de nouvelles voies thérapeutiques essayant de contrôler la réaction inflammatoire, les métalloprotéinases macrophagiques ou l'apoptose des cellules musculaires lisses, se développeront et permettront de mieux contrôler la vulnérabilité des plaques athéroscléreuses.

II apparaît ainsi qu'à chaque étape de l'évolution de l'athérosclérose, le clinicien doit répondre à un double pari, celui de la détection précoce et celui du traitement adapté. L'analyse des progrès actuels, notamment sur le plan thérapeutique, prouvent clairement que les solutions ne peuvent venir que de l'analyse approfondie des mécanismes cellulaires contrôlant les différentes étapes. Les nouvelles approches diagnostics et thérapeutiques de l'athérosclérose apparaissent comme un défi commun du biologiste et du clinicien

\section{RÉFERENCES}

1. Resnick N, Yahav H, Schubert S, Wolfovitz $E$, Shay A. Signalling pathways in vascular endothelium activated by shear stress : relevance to atherosclerosis. Curr 0 pin Lipidol 2000 ; 11 : 167-77.
2. Allen S. Lipid/ vascular wall inter
curr O pin Cardiol $1998 ; 13: 439-446$.
3. Kataoka H, Kume N, Miyamoto S, et al. Expression of lectinlike oxidized low-density lipoprotein receptor-1 in human atherosclerotic lesions. Circulation $1999 ; 99$ : 3110-7.

4. Schultz D, Harrison DG. Quest for fire : seeking the source of pathogenic oxygen radicals in atherosclerosis. Arterioscler Thromb Vasc Biol $2000 ; 20$ : 1412-3.

5. Memon R, Straprans I, Noor M, Holleran W, Uchida $Y$, Moser A. Infection and inflammation induce LDL oxidation in vivo. A rterioscler Thromb Vasc Biol $2000 ; 20$ : 153642.

6. TeplyakovAI, Pryschepova EV, Kruchinsky NG, Chegerova TI. Cytokines and soluble cell adhesion molecules. Possible markers of inflammatory response in atherosclerosis. Ann NY Acad Sci $2000 ; 902$ : 320-2.

7. Aggoun Y, Bonnet D, Sidi D, et al. Arterial mechanical changesin children with familial hypercholesterolemia. Arterioscler Thromb Vasc Biol $2000 ; 20: 2070-5$.

8. Prasad A, Narayanan S, Husain S, Padder F, Waclawiw M, Epstein N, Quyyumi AA. Insertion-deletion polymorphism of the ACE gene modulates reversibility of endothelial dysfunction with $A C E$ inhibition. Circulation $2000 ; 102$ : 35-41

9. Bandoh T, Mitani H, Niihashi M, et al. Fluvastatin suppresses atherosclerotic progression, mediated through its inhibitory effect on endothelial dysfunction, lipid peroxidation, and macrophage deposition. I Cardiovasc Pharmacol $2000 ; 35$ : 136-44.

9. Duplaa C, Couffinhal T, Labat L, et al. Monocyte-macrophage recruitment and endothelial adhesion protein expression in human atherosclerotic lesions. A therosclerosis 1996 ; 121 : 253-66.

10. Dong ZM, Brown AA, Wagner DD. Prominent role of $P$-selectin in the development of advanced atherosclerosis in ApoE-deficient mice. Circulation $2000 ; 101$ : 2290-5.

11. Mach F, Schonbeck U, Sukhova G, Atkinson $E$, Libby P. Reduction of atherosclerosis in mice by inhibition of CD40 signalling. N ature 1998 ; 394 : 200-3.

12. Frostegard J, Ulfgren AK, Nyberg $P, \mathrm{He}$ din U, Swedenborg J, Andersson U, H ansson GK. Cytokine expression in advanced human atherosclerotic plaques: dominance of pro-inflammatory (Th1) and macrophagestimulating cytokines. A therosclerosis 1999; $145: 33-43$.

13. Mallat Z, Besnard S, Duriez M et al. Protective role of interleukin-10 in atherosclerosis Circ Res 1999 ; 85 : e17-24.

14. Benditt EP, Benditt JM. Evidence for a monoclonal origin of human atherosclerotic plaques. Proc Natl Acad Sci USA 1973; 70 : 1753-6.

15. Clowes AW, Schwartz SM. Significance of quiescent smooth muscle migration in the injured rat carotid artery. Circ Res 1985; 56 : 139-45.
16. Salomaa V, Matei C, Aleksic N, et al. Soluble thrombomodulin as a predictor of incident coronary heart disease and symptomless carotid artery atherosclerosis in the Atherosclerosis Risk in Communities (ARIC) Study: a case-cohort study. Lancet $1999 ; 353$ : 1729-34.

17. O ish Y Y, Wakatsuki T, Nishikado A, O ki T, Ito $S$. Circulating adhesion molecules and severity of coronary atherosclerosis. Coron A rte ry Dis $2000 ; 11: 77-81$.

18. Paiker JE, Raal FJ, von Arb M. Auto-antibodies against oxidized LDL as a marker of coronary artery disease in patients with familial hypercholesterolaemia. Ann Clin Biochem $2000 ; 37: 174-8$.

19. Sablotzki A, Welters I, Lehmann N, Menges T, Gorlach G, Dehne M, Hempelmann G. Plasma levels of immunoinhibitory cytokines interleukin-10 and transforming growth factor-beta in patients undergoing coronary artery bypass grafting. Eur J Cardiothorac Surg $1997 ; 11:$ 763-8.

20. Allan PL, Mowbray PI, Lee AJ, Fowkes FG. Relationship between carotid intimamedia thickness and symptomatic and asymptomatic peripheral arterial disease. The Edinburgh Artery Study. Stroke 1997 ; $28: 348-53$

21. Boquist $S$, Ruotolo $G$, Tang R, et al. Alimentary lipemia, postprandial triglyceriderich lipoproteins, and common carotid intima-media thickness in healthy, middle-aged men. Circulation $1999 ; 100$ : 723-8.

22. Davies M, Richardson $P$, Woolf $N$ et al. Risk of thrombosis in human atherosclerotic plaques: Role of extracellular lipid, macrophage, and sommoth muscle cell content. $\mathrm{Br}$ H eart ] $1993 ; 69$ : 377-81.

23. Crawley J, Lupu F, Westmuckett AD, Severs NJ, Kakkar VV, Lupu C. Expression, localization, and activity of tissue factor pathway inhibitor in normal and atherosclerotic human vessels. Arterioscler Thromb Vasc Biol $2000 ; 20: 1362-73$.

24. Mallat Z, Hugel B, O han J, Leseche G, Freyssinet JM, Tedgui $A$. Shed membrane microparticles with procoagulant potential in human atherosclerotic plaques: a role for apoptosis in plaque thrombogenicity. Circulation 1999 ; 99 : 348-53.

25. Tedgui A, Mallat Z. Smooth muscle cells: another source of tissue factor-containing microparticles in atherothrombosis ? Circ R es $2000 ; 87: 81-2$.

\section{TIRÉS À PART}

J. Bonnet. 